\title{
Philosophiques
}

\section{La théorie hégélienne du savoir}

\section{Laurent-Paul Luc}

Volume 7, numéro 1, avril 1980

URI : https://id.erudit.org/iderudit/203131ar

DOI : https://doi.org/10.7202/203131ar

Aller au sommaire du numéro

Éditeur(s)

Société de philosophie du Québec

ISSN

0316-2923 (imprimé)

1492-1391 (numérique)

Découvrir la revue

Citer cet article

Luc, L.-P. (1980). La théorie hégélienne du savoir. Philosophiques, 7(1), 55-76.

https://doi.org/10.7202/203131ar d'utilisation que vous pouvez consulter en ligne.

https://apropos.erudit.org/fr/usagers/politique-dutilisation/ 


\title{
LA THÉORIE HÉGÉLIENNE DU SAVOIR* par Laurent-Paul Luc
}

\author{
SIGLES ET EDITIONS UTILISES :
}

N.

Theolngiscbe Jugendscbriften. Reproduction photomécanique par Minerva, Frankfurt, 1966.

T.F.

Tübinger Fragment.

G.C.S.

Der Geist des Christentums und sein Schicksal.

1792-1793 NOHL

1798-1799 NOHL

LEsprit da Christianisme et son destin, trad. Martin, Paris, Vrin, 1948. Cité E.C.D.

S.F.

System Fragment ton 1800.

Differenz

Differenz des Fibbtescben und Schellingschen Systems der Pbilnsopbie, in Jeaner Kritische Schriften.

Différence des systemes philosopbiques de Fichte et de Schelling, in Premières publications, trad. Méry, Paris, Vrin, 1952. Cité : Différence.

G.W.

Glauben und Wissen, in Jeaner Kritische Schriften.

Foi et Savoir, in Premieres publications, trad. Méry. Ciré : F.S.

Ph.G.

Phänomenolugie des Geistes.

La Pbénoménologie ¿ IEsprit, trad. Hyppolite, Paris, Aubir, 1939-1941, 2 vol. Cité : Ph.E.

$W . L$

Wissenschaft der Logik.

1801

MEINER

MEINER

1807

MEINER

1812-1816 MEINER

Sizence de la Logique, trad. Jankélévitch, Paris, Aubier, 1947-1949. 4 vol. Cité: S.L.

* Ce texte est celui d'une communication présentée au cours collectif "Théories de la connaissance de Platon a Piaget ", organisé à la session d'automne 1979 par le Département de Philosophie de l'Université de Sherbrooke. 
Enz.

MEINER

$1827-1830$

Enzyklopädie der philosophischen Wissenschaften. Encyclopédie des scienses philosopbiques. I. La science de la logique, trad. Bourgeois, Paris, Vrin, 1970. Cité : Enc.

Encyclopédie des sciences philnsophiques en abregé, trad. Gandillac, Paris, Gallimard, 1970. Cité : Enc. (Gandillac).

Ph.R.

Grundlinien der Philosopbie des Rechtes.

MEINER

Principes de la philosopbie du droit, trad. Derathé, Paris, Vrin, 1975. Cité : Ph.D.

Vorlesungen

V.Pb.R

FROMMAN

Vorlesungen über die Philosopbie der Religion.

Leģons sur la pbilosopbie de la religion, trad. Gibelin, Paris, Vrin, 1954-1959, 5 vol. Cité : L.Ph.R.

G.Ph.

FROMMAN

Vorlesungen über die Geschichte der Philosophie.

Leşons sur l'bistnire de la pbilosopbie.

Introduction: Systeme et bistoire de la pbilosophie, trad. Gibelin, Paris, Gallimard, 1954. Cité : L.H.Ph. Introduction.

Legons sur l'bistnire de la philosophie, trad. Garniron, Paris, Vrin, 1971- . 7 vol. 4 parus. Cité : L.H.Ph.

Briefe

Briefe won und an Hegel, 4 vol.

Correspondance (1785-1822), trad. Carrère, Paris, Gallimard, 1962-1963. 3 vol. Cité : Correspondance. 
Dans le champ de la pensée essentielle, toute contre-position (Widerlegen) est sottise. La lutte entre les penseurs est la « lutte amoureuse ", lutte qui est celle de la chose même.

Heidegger ${ }^{1}$

Merleau-Ponty, qui avait l'habitude d'accueillir les pensées des philosophes moins comme des pensées que comme des appels à penser, a dit de Hegel qu'il " est à l'origine de tout ce qui s'est fait de grand en philosophie depuis un siècle - par exemple du marxisme, de Nietzsche, de la phénoménologie et de l'existentialisme allemand, de la psychanalyse ». Il ajoutait un peu plus loin : "On pourrait dire sans paradoxe que donner une interprétation de Hegel, c'est prendre position sur tous les problèmes philosophiques, politiques et religieux de notre siècle ${ }^{2}$. A Affirmations étonnantes sans doute déjà inspirées, comme le suggèrent les dernières Notes de travail ${ }^{3}$, par la recherche d'une "bonne dialectique " qui, loin de s'installer dans "la jonction d'un sujet, de l'être et des autres sujets ${ }^{4}$, invite à la pratique d'une "hyperdialectique ${ }^{5}$ ", située "hors de la philosophie du sujet et de l'objet" ". On sait que la mort vint interrompre cet effort de Merleau-Ponty dont le chemin de pensée a été tracé par ce que Heidegger appelait, lors des célèbres «Séminaires du Thor », la nécessité

1. Heidegger, M., Lettre sur l'bumanisme, trad. Munier, Paris, Aubier, 1964, p. 90-91.

2. Merleau-Ponty, M., "L'existentialisme chez Hegel», in Sens et Non Sens, Paris, Nagel, 1966 , p. 109 , p. 110.

3. Merleau-Ponty, M., Le Visible et l'Invisible, suivi de Notes de travail, Paris, Gallimard, 1973.

4. Merleau-Ponty, M., Les aventures de la dialectique, Paris, Gallimard, Idées, $n^{\circ} 375,1977$, p. 297.

5. «La mauvaise dialectique commence presque avec le dialectique, et il n'est de bonne dialectique que celle qui se critique elle-même et se dépasse comme énoncé séparé ; il n'est de bonne dialectique que l'hyperdialectique. "(Le Visible et l'Invisible, p. 129).

6. Notes de tratiall, p. 260. 
d'« entrer en débat avec Hegel ${ }^{7}$, nécessité qui paraît contraindre mêtme ceux qui ne sont pas enclins à partager la prétention démesurée d'un savoir absolu - encore moins du savoir absolu hégélien - à s'abriter sous l'ombre de celui qui, en 1807, a cru que tout ce qui a été vécu et pensé par l'humanité s'exhibe dans l'ouvrage sans doute le plus énigmatique de l'histoire de la philosophie: la Phénoménologie de l'Esprit.

\section{L'APOLOGIE DE LA CONNAISSANCE RATIONNELLE}

On sait que la Phénoménologie de l'Esprit, rédigée en peu de temps à Iéna ${ }^{9}$, veut conférer à la philosophie un autre nom que celui qu'on lui reconnaissait jusque-là : "Collaborer à ce que la philosophie se rapproche de la forme de la Science, - du but [qui est] de pouvoir déposer son nom d'amour pour le savoir et à être savoir effectif, - c'est là ce que je me suis proposé ${ }^{10}$. » Cette métamorphose, Hegel a cru l'accomplir en présentant la description point par point de tous les phénomènes de savoir historiquement et logiquement appelés (et élus) par l" "esprit du monde (Weltgeist) » qui, après avoir eu « la patience de parcourir ces formes dans toute l'extension du temps et d'entreprendre le prodigieux labeur de l'histoire universelle " ${ }^{11}$, accède maintenant, grâce à la "Ré-Intériorisation (Er-Innerung) " 12 opérée dans l'« histoire conçue (begriffne Geschichte) ${ }^{13}$, à sa pure nomination conceptuelle célébrée dans la Science de la Logique.

7. Heidegger, M., "Les Séminaires du Thor ", in Questions IV, Paris, Gallimard, 1976, p. 213 .

8. Le caractère énigmatique de cetre ceuvre apparaît déjà à travers les modifications de son titre. Lors de sa publication en 1807, l'ouvrage s'intitule : Sizence de l'Expérience de la Conscience. En cours d'impression, ce titre devient : Science de la Phénoménologie de l'Esprit. Un troisième titre surgit lorsque l'ouvre parait un an après la mort de Hegel: Phénoménologie de l'Esprit.

9. D'octobre 1805 à janvier 1807.

10. Ph.G., $12 ;$ Ph.E., 1, p. 8 . Traduction modifiée.

11. Ph.G., $27 ;$ Ph.E., I, p. 27.

12. Ph.G., $564 ; P h . E, 11$, p. 312 . Traduction modifiée.

13. Ph.G., $564 ;$ Ph.E., II, p. 313. 
Qu'en est-il de cette « entrée en scène (Auftreten) » de la Science, dont Hegel nous dit qu'elle est "la Chose même (die Sache selbst) " à laquelle on doit en venir dans la philosophie, à « savoir le connaître effectif de ce qui est en vérité » ${ }^{14}$ et dont il s'est efforcé d'encercler la scientificité dans l'Encyclopédie des sciences philosophiques? Ayant cru comprendre que c'est cette question que je devais aborder pour traiter du « problème de la connaissance » dans la philosophie hégélienne, j'ai préféré, plutôt que de l'affronter directement en m'aventurant dans le problème des difficiles rapports de la Phénoménologie de l'Esprit à la Science de la Logique ou en parcourant les cercles en-cyclo-pédiques, y préparer en explorant avec vous quelques-unes des questions que m'a suggérées la métaphore qui ouvre « La période de l'entendement pensant (denkenden Verstandes)» dans les Legons sur l'bistoire de la philosopbie :

Avec Descartes nous entrons à proprement parler dans une philosophie autonome qui sait qu'elle vient de la raison autonome et que la conscience-de-soi est [un] moment essentiel du vrai. Ici, pouvons-nous dire, nous sommes chez-nous (sind wir zu hause), et [nous] pouvons, comme le navigateur après [un] long périple sur la mer démontée crier «terre ${ }^{15}$.

Cette métaphore, d'une écriture assez enthousiaste, nous indique au moins deux vocations, deux « affaires » inséparables, du moins pour Hegel :

1) l'affaire du discours philosophique comme tel,

2) l'affaire du discours hégélien lui-même.

Affaires inséparables, parce qu'en étant, comme on l'a dit, le seul penseur de l'Occident à avoir fait "l'expérience pensante de l'histoire de la pensée ${ }^{16}$, Hegel n'a voulu être rien d'autre que celui qui recueille la vérité déposée dans l'histoire de la philosophie. Vérité déposée, non seulement reflétée, parce que le discours hégélien ne se présente pas comme un discours sur l'histoire, mais comme le discours de l'histoire en lequel l'histoire de la philosophie, loin de «détailler la collection des opinions philosophiques comme

14. Ph.G., 63 ; Ph.E., p. 65. Traduction modifiée.

15. G.Ph., III, 328 .

16. Heidegger, M., "La parole d'Anaximandre ", in Chemins qui ne mènent nulle part, Paris, Gallimard, 1962, p. 263. Traduction modifiée. 
elles se sont produites et présentées dans le temps ${ }^{17}$, présente " la galerie des héros de la raison pensante (denkenden Vernunft), qui, par la vertu de cette raison, ont pénétré dans l'essence des choses, de la nature et de l'esprit, dans l'essence de Dieu, et nous ont acquis par leur effort le trésor suprême, celui de la connaissance rationnelle (Vernunfterkenntniss) ${ }^{18}$ ».

Qu'est-ce donc que cette " connaissance rationnelle» dont la Préface des Grandes lignes de la Philosopbie du Droit nous assure qu'elle procure une "paix (. . .) chaleureuse avec la réalité $^{19}$ *?

Interrogation sans doute paradoxale, puisque à la poser à Hegel - surtout si on désire par là limiter sa pensée à une théorie de la connaissance - on risque de s'enfoncer dans un malentendu. On sait que les questions qui ont motivé la constitution d'une théorie de la connaissance sont nées de cette époque récente où la philosophie allemande, après avoir considéré le projet kantien d'établir la scientificité de la métaphysique comme une rêverie qui se serait effondrée sous le positivisme, s'est mise en frais, même divisée par le psychologisme, le néo-kantisme et la phénoménologie, de s'enquérir, en réaction à la spéculation absolue de Hegel, d'une théorie dite de la connaissance qui aurait pour tâche de franchir ce que Husserl appelait les «abîmes de difficultés ${ }^{20}$ creusés par la possibilité de la connaissance. Que la pensée de Hegel ait été étrangère à une telle perplexité qui, d'après Heidegger, « aujourd'hui encore passe souvent pour l'affaire essentielle de la philosophie ${ }^{21}$, c'est ce que révèle déjà l'itinéraire qu'il a suivi de Tübingen à Iéna.

17. G.Pb., I, $39 ;$ L.H.Pb.. Introduction, p. 32. Traduction modifiée.

18. G.Pb., I, $27 ;$ L.H.Ph.. Introduction, p. 29.

19. Ph.R., $17 ;$ Ph.D., p. 58 . Traduction modifiée.

20. Husser1, E., Lidée de la phénoménolngie, trad. Lowitt, Paris, P.U.F., 1978, p. 39

21. Heidegger, M., "Moira (Parménide, VIII, 34-41)», in Essais et Conférences, trad. Préau, Paris, Gallimard, 1978, p. 282. 


\section{L'ITINÉRAIRE HÉGÉLIEN}

Une lecture attentive des Jugendschriften (octobre 1792 janvier 1801) ne nous met pas en présence d'une méditation tourmentée par la perplexité quant à la possibilité de la connaissance. Même tournée, lors des années de formation au Stift de Tübingen, vers l'élaboration d'un projet d'éducation populaire à la liberté, ou séduite à Berne par l'idéal révolutionnaire, ou tentée à Frankfurt de désavouer la philosophie au profit de la religion, la recherche du jeune Hegel demeure fascinée par une pensée de la vie, qui est identiquement une pensée de la manifestation où se met intégralement en vue la totalité de ce qui est. C'est bien la quête d'une unité fondatrice permettant à l'homme, enfin initié à la convenance des événements, d'être "tout à fait chez soi (ganz einheimisch) ${ }^{22}$ dans tout ce qui advient, que veut étayer le questionnement du Tübinger Fragment tout entier soucieux de "parler (. . ) in concreto ${ }^{23}$ du sens de la vie religieuse et politique ; c'est bien la volonté de ne vivre que pour la « libre vérité ${ }^{24}$, qui suscite les écrits bernois; c'est bien la voie d'accès au «Tout de la vie ${ }^{25}$ que cherche à préparer la critique francfortoise de toute réflexion impuissante à surmonter l'opposition "du pensant et du pensé » ${ }^{26}$. "Conciliation et être, lit-on dans la $V^{e}$ ébauche préparatoire à l'Esprit $d u$ Christianisme et son destin, ont le même sens ${ }^{27}$. "Ce qui se fait entendre dans cette formule, c'est encore la volonté d'élaborer une "théorie du savoir " qui permettrait de "penser la pure vie ${ }^{28}$, c'est-à-dire de penser l'Être — puisque, pour Hegel, «Pure vie est être ${ }^{29}$ - dans une ultime adéquation de la différence Vie-vivant, Être-étant.

Enracinés dans la conviction que le destin des choses est de se livrer intégralement à une pensée présente à soi, les travaux d'Iéna établiront que c'est au-delà du droit, de l'art et

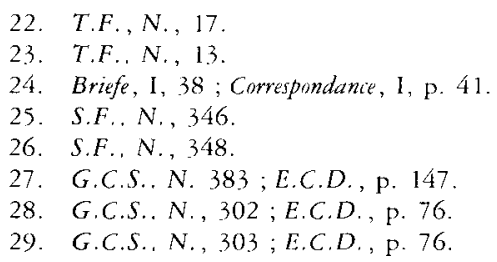


de la religion, à la philosophie seule qu'il appartient d'accomplir cette tâche par la mise en œuvre de l'activité systématique et spéculative de la raison.

Si les époques de jeunesse (1792-1801) et de maturation (1801-1807) échappent à toute perplexité quant à la cohérence de la pensée avec elle-même ou quant aux possibilités d'une cohérence de la pensée avec l'être, l'époque de maturité confie la prise en compte du " connaitre effectif de ce qui est en vérité » à la «positivité » des sciences ainsi codifiées en raison de leur champ d'investigation délimité par la sphère des phénomènes contingents, et à l'«effectivité " de la pensée ${ }^{30}$ qui déploie la pure intelligibilité du réel grâce à la « sursomption (Aufhebung) ${ }^{31}$ de la double opposition qui caractérise le registre de la conscience : celle de la certitude et de la vérité, celle du sujet et de l'objet. Dès le début d'Iéna, cette entreprise spécifie l'unique savoir de la philosophie:

... on ne doit pas (. . .) admettre que la tâche de la vraie philosophie est de résoudre, en les envoyant terme à terme, les oppositions qui se présentent et sont conçues comme celles tour à tour de l'esprit et du monde, du corps et de l'âme, du Moi et de la Nature, etc. ; au contraite son unique idée qui a pour la philosophie réalité et véritable objectivité est l'êtresursumé absolu de l'opposition, et cette identité absolue n'est pas un postulat universel, subjectif, irréalisable, -- elle est au contraire la seule réalité véritable, - et la connaissance de cette identité n'est pas une foi, c'est à-dire un au-delà pour le savoir, elle est au contraire l'unique savoir de la philosophie ${ }^{32}$.

Parmi les textes innombrables qui n'hésitent pas à frayer avec l'effroi provoqué par les «abîmes de difficultés » quant à la possibilité de la connaissance, retenons, en plus de la fameuse formule proclamant l'identité du réel et du ration-

30. Enz., \#9, 42 ; Enc., p. 173

31. Le néologisme "sursomption" a été suggéré par Y. Gauthier. "Nous proposons, écrit-il, la traduction "sursumer " et "sursomption" pour "aufheben " et "Aufhebung ". La dérivation étymologique s'appuie sur le modèle « assumer - assomption ». La sémantique du mot correspond à l'antonyme de "subsomption " que l'on trouve chez Kant. La sursomption définit donc unc opération contraire à celle de la subsomption, qui consiste à poser la partie dans ou sous la totalité ; la sursomption, l' "Aufhebung", désigne le procès de la totalisation de la partie. Voir là-dessus la Logique dléna. " ("Logique hégélienne et formalisation", in Dialogue, Revue canadicnne de philosophie, septembre 1967, p. 152, note 5).

32. G.W., $325 ;$ F.S., p. 205-206. Traduction modifiée. 
nel ${ }^{33}$, les trois textes suivants où se condense la subversion hégélienne des conceptions très traditionnelles de la connaissance.

Mais l'examen de la connaissance ne peut se faire autrement qu'en connaissant; dans le cas de ce prétendu instrument, l'examiner ne signifie rien d'autre que le connaître. Mais vouloir connaitre avant de connaitre est aussi absurde que le sage projet qu'avait ce scolastique, d'apprendre à nager avant de se risquer dans l'eau ${ }^{34}$.

Ce qu'il y a entre la raison comme esprit conscient-de-soi et la raison comme réalité présente (als vorhandener Wirklichkeit), ce qui sépare la première de la seconde et empêche de trouver l'apaisement en celle-ci, c'est la chaîne de quelque abstraction qui ne s'est pas libérée pour atteindre le concept. Reconnaître la raison comme la rose dans la croix du présent et par là se réjouir de ce présent, cette vision rationnelle est la réconciliation (Versöbnung) avec la réalité, réconciliation que procure la philosophie à ceux à qui est apparue une fois l'exigence intérieure de concevoir (begreifen) et de conserver dans ce qui est substantiel la liberté subjective et de ne pas s'en tenir de même avec cette liberté subjective au particulier et au contingent, bien plutôt à ce qui est en soi et pour soi ${ }^{35}$.

C'est une représentation naturelle que, avant que l'on en vienne, dans la philosophie, à la Chose même, savoir au connaître effectif de ce qui est en vérité, il serait nécessaire de s'entendre auparavant sur la connaissance qu'on considère comme l'instrument à l'aide duquel on s'empare de l'absolu ou comme le moyen grâce auquel on l'aperçoit. Une telle préoccupation semble justifiée, en partie, parce qu'il pourrait y avoir diverses espèces de connaissances, et que dans le nombre l'une pourrait être mieux adaptée que l'autre pour atteindre ce but final, - justifiée donc aussi par la possibilité d'un choix erroné parmi elles, - en partie aussi parce que la connaissance étant une faculté d'une espèce et d'une portée déterminée, sans détermination plus précise de sa nature t de ses limites, on peut rencontrer les nuées de l'erreur au lieu d'atteindre le ciel de la vérité. À la fin, cette préoccupation doit se transformer en la conviction que toute l'entreprise de gagner à la conscience ce qui est en soi par la connaissance est dans son concept un

33. "Was ternünftigt ist. das ist wirklich : und uas uirklich ist, das ist vernünfig."

(Ph.R., 14)

34. Enz., \#10, 42 ; Eni., p. 175.

35. Ph.R., 16;Ph.D., p. 57-58. Traduction modifiée. 
contre-sens, et qu'il y a entre la connaissance et l'absolu une ligne de démarcation très nette ${ }^{36}$.

Le contexte auquel renvoient ces trois textes est celui de la définition d'une forme de philosopher opposée à une forme spécifiquement moderne inaugurée selon Hegel par Descartes. Rappelons la VIII ${ }^{\mathrm{e}}$ des Règles pour la direction de l'esprit :

Si quelqu'un se propose comme problème d'examiner toutes les vérités pour la connaissance desquelles la raison humaine suffit (examen que doivent faire, ce me semble, au moins une fois dans la vie, tous ceux qui s'efforcent sérieusement de parvenir à la sagesse), il trouvera certainement par les règles qui ont été données, que rien ne peut être connu avant l'entendement, puisque la connaissance de toutes les autres choses en dépend, et non l'inverse ${ }^{37}$.

Il n'en va pas autrement chez Hume ${ }^{38}$ et chez Kant pour qui « instituer un tribunal » qui garantisse la raison « dans ses prétentions légitimes » réalise le vœu "d'un siècle qui ne veut pas se laisser bercer plus longtemps par une apparence de savoir $\gg^{39}$.

S'il fallait repérer dans son origine historique le sens de la véritable base de cette conception moderne de la connaissance, il conviendrait sans doute de se reporter à l'œuvre de Galilée qui a été comme réfléchie dans les Règles pour la direction de l'esprit. Cet ouvrage de méthodologie veut nous apprendre que «le but des études (entendons du savoir) doit être de diriger l'esprit pour qu'il porte des jugements solides et vrais sur tout ce qui se présente à lui ${ }^{40}$. Cette formulation nous plonge d'emblée dans un contexte moderne pour autant que « sauver les phénomènes », ce n'est plus prêter attention à ce qui se manifeste de soi-même mais projeter idéalement sur ce qui est

36. Ph.G., 63 ; Ph.E., p. 65. Traduction modifiée.

37. Descarres, R., "Règles pour la direction de l'esprit ", in Oeuvres et lettres, Paris, N.R.F., La Pléiade, 1970, p. 63.

38. "It is evident, that all the scicnces have a relation, greater of less, to human nature; and that, however wide any of them may seem to run from it, they still return back by one passage or another. Even Mathematio. Natural Philosophy, and Natural Religion, are in some measure dependent on the science of MAN ; since they lie under the cognisance of men, and are judged of by their powers and faculties. "Hume, D., A Treatise of Human Nature, New York, Everyman's Library, 1968, Vol. I, p. 4.

39. Kant, E., Critique de la Raisun Pure, trad. Tremesaygues et Pacaud, Paris, P.U.F., 1965, p. 6-7.

40. Descartes, R., op. cit., p. 37. 
- idéalement, c'est-à-dire en vertu d'une sorte de purification de ce qui se donne dans le monde sensible - la lumière de la conscience ou de l'esprit, qui non seulement convoque toutes choses à apparaître devant elle mais décide de la manière dont les choses doivent se présenter. Ce qui est, c'est ce qui apparaît dans le cadre d'une idéalisation pratiquée par l'esprit ; ce qui est, c'est ce qui apparaît eu égard à une anticipation qui est l'acte du Cogito.

Lorsqu'il proclame qu'avec Descartes la philosophie peut enfin «crier terre", c'est cette mutation de l'idée de phénomène que Hegel veut mettre en lumière, mutation dont il se fera lui-même l'héritier et qu'il bouleversera à son tour en surmontant l'hétérogénéité des trois idées kantiennes de la raison: Dieu, le Monde et l'Âme ${ }^{41}$, grâce au principe idéaliste de la philosophie :

Toute philosophie est essentiellement idéalisme ou bien a celui-ci au moins à son principe, et la question est donc seulement de savoir jusqu'à quel point ce principe est effectivement mis en application ${ }^{42}$.

Que peut bien signifier cet idéalisme associé à l'"être chez soi » qui, selon Hegel, définit la philosophie: "la philosophie est précisément cela : être vraiment chez soi (bei sich zu hause zu sein), - que l'homme soit dans son esprit chez lui en terre natale (in seinem Geiste zu hause sei, heimathlich bei sich $)^{43} »$.

\section{L'ÊTRE CHEZ SOI : MÉDITATION, NÉGATIVITÉ, DIALECTIQUE}

Se trouver chez soi évoque d'abord l'idée d'une autonomie, d'une souveraineté, d'une absolution de toute altérité. Affirmer de la philosophie qu'elle est autonome, souveraine,

41. Ce surmontement est opéré dans la sysrématique encyclopédique de l'Absolu qu'expose l'articulation organique des trois syllogismes de la philosophie : Logique - Nature Esprit, Nature - Esprit - Logique, Esprit - Logique - Nature. Enz. \#574-577, 462-463; Enc. (Gandillac), p. 499-500.

42. W.L., II, $145 ;$ S.L., I, p. 158. Traduction modifiée.

43. G.Ph., I, $190 ;$ L.H.Ph., I, p. 23. 
absolue, ce n'est pourtant pas l'enrouler sur elle-même : elle prendrait alors la forme d'un savoir qui laisserait subsister d'autres savoirs (y compris celui du non-savoir) à côté d'elle. Dans ce cas, la philosophie obéirait bien à ses propres lois, elle serait autonome et absolue, mais il s'agirait d'une autonomie abstraite, d'une absoluité relative. La philosophie entre réellement chez soi, elle accède à une autonomie réelle, à une absoluité absolue seulement au moment où elle parvient à intégrer toutes les formes d'expérience du réel en montrant qu'elle est elle-même appelée par tous les savoirs.

La métaphore de la «terre natale», découverte par Descartes, suggère que cette autonomie, la philosophie l'acquiert dans la «conscience de soi » qui est comme le sol de toutes les figures du savoir et de l'étant en totalité, sol qu'Héraclite le premier a foulé sans pourtant l'avoir arpenté ${ }^{44}$. Cette autonomie, cette absoluité, cette entrée dans le Soi de la philosophie, l'image du navigateur nous invite à la penser comme un mouvement, comme une ré-flexion :

1) l'arrivée dans la patrie résulte d'un mouvement où l'on n'était pas encore chez soi,

2) l'entrée dans le Soi présuppose une sorte d'errance, une sorte de déchirement ou de scission (la mer démontée).

La Préface de la Phénoménologie de l'Esprit souligne que ce mouvement n'appartient pas à un champ délimité d'investigation, mais est intimement lié à la vocation ou à l'affaire de la pensée elle-même :

La substance vivante est l'être qui est stijet en vérité ou, ce qui signifie la même chose, est l'être qui est effectivement réel en vérité, mais seulement en tant que cette substance est le mouvement de se-poser-soi-même (Sichselbstsetzens), ou est la médiation entre son propre devenir-autre et soi-même. Comme sujet, elle est la pure et simple négativité ; c'est pourquoi elle est la scission (Entzweiung) du simple en deux parties, ou la duplication opposante, qui, à son tour, est la négation de cette diversité indifférente et de son opposition; c'est seulement

44. "C'est (...) chez Héraclite qu'on doit rencontrer pour la première fois l'idée philosophique sous sa forme spéculative (. . .). Ici nous voyons la terre (Hier sehen wir Land). »G.Pb. , 1, $344 ;$ L.H.Ph. , 1, p. 154. Traduction modifiée. 
cette égalité se reconstituant ou la réflexion en soi-même dans l'être-autre qui est le vrai - et non une unité originaire comme telle, ou une unité immédiate comme telle. Le vrai est le devenir de soi-même, le cercle qui présuppose et a au commencement sa propre fin comme son but, et qui est effectivement réel seulement moyennant son actualisation développée et moyennant sa $\mathrm{fin}^{45}$.

Ce texte donne à entendre que « le mouvement de naître et de périr, mouvement qui lui-même ni ne naît ni ne périt ${ }^{46}$, mouvement qui est l'Apparition (Erscheinung) de l'Absolu, se présente comme un mouvement circulaire que circonscrivent les notions de médiation, de négativité et de dialectique. Tentons de préciser chacune de ces notions.

Il convient d'abord de noter que l'autoposition (Sichselbst-setzen) dont parle Hegel signifie que la réalité - dont le destin est d'être conscience de soi - s'avère au cours d'un procès qui est à la fois division et sursomption de cette division. Entendu « non comme substance, mais précisément aussi comme sujet ${ }^{47}$, le Vrai est ce mouvement de se-posersoi-même à travers un déchirement de sa simplicité initiale. Il s'agit d'un mouvement pour ainsi dire en repos qui se déroule à la manière d'un processus de médiation où c'est l'Être lui-même qui est médiateur entre son devenir-autre et soimême. La médiation ne désigne pas ici une instance témoin extérieure aux parties opposées, elle ne réfere pas à un mécanisme extérieur d'arrangement mais se révèle bien plutôt comme anéantissement et surgissement où le sujet se saisit lui-même comme identité du même et de l'autre : c'est le sujet lui-même qui se devient autre en tant qu'objet et qui, à travers cette altérité, se constitue lui-même identité du sujet et de l'objet.

Ce procès peut être appelé négativité, puisque, au sein de ce mouvement, il y a négation d'une simplicité primitive, opposition de chacun des termes à l'égard de l'autre et négation de cette opposition dans le but d'arriver à une égalité de soi avec soi. Ainsi, cette égalité, ou plutôt cette égalisation,

45. Pb.G., $20 ;$ Pb.E., I, P. $17-18$.

46. Pb.G., $39 ;$ Ph.E., I, p. 40.

47. Ph.G., 19;Pb.E., I, p. 17. 
est-elle devenir de soi à l'intérieur de soi ou, comme l'écrit Hegel, "l'égalité-avec-soi-même se mouvant (die sich bewegende Sichselbstgleichheit) " ${ }^{48}$ pour autant qu'en s'engendrant elle-même à partir de son arrachement à elle-même, elle retourne à son immédiateté première, mais comme à sa richesse pleinement manifestée.

Ce procès, Hegel en voit une illustration dans le fruit, mort de la fleur ${ }^{49}$. L'autonégation de la belle immédiateté primitive de la floraison est l'occasion d'accéder à la maturité qui est dès lors un retour à soi, puisqu'il y aura une nouvelle plante. Mais ce retour naturel n'obéit pas parfaitement à cette circularité : la réduplication est bien en soi, mais ne devient pas pour soi. C'est que la vie ne s'accomplit pas dans l'ordre organique, mais dans l'ordre spirituel :

Le fruit, la graine n'est pas pour le premier germe, bien plutôt seulement pour nous; dans l'esprit, les deux aspects ne sont pas seulement en soi la même nature, mais c'est un un-pourl'autre, et justement en cela un être-pour-soi. Ce pour quoi l'autre est, est la même chose que l'autre. [C'est] seulement par là [que] l'esprit est chez soi-même dans son autre. L'évolution de l'esprit est sortie-hors-de-soi, déploiement-de-soi, et en même temps retour-à-soi ${ }^{50}$.

Ce « retour à soi » peut être nommé dialectique dans la mesure où dans son autoposition le sujet se rassemble ou se recueille $(\lambda \epsilon \gamma \epsilon \omega v)$ lui-même comme sujet accompli à travers $\left(\delta\llcorner\alpha)\right.$ sa duplication opposante $\mathrm{e}^{51}$.

48. Ph.G., $21 ;$ Pb.E., I, p. 19.

49. Ph.G., $10 ;$ Ph.E., 1, p. 6.

50. G.Ph., I, $51 ;$ L.H.Ph. Introduction, P. 102. Traducrion modifiée.

51. Le dernier chapitre de la Logique du Concept précise que « si l'on tient absolument à compter (. . .), la forme abstraite (de la dialectique) peut être considérée comme une quadruplicité au lieu de la triplicité " (W.L., II, 498 ; S.L., IV, p. 564. Traduction modifiée). Le procès dialectique se déroule comme un mouvement à 4 remps (immédiateté première, négation, négation de la négation, immédiateté devenue) qui échappe au schéma triadique (thèse, antithèse, synthèse) adapté à la pensée formelle abstraite. L'illustration la plus éclairante de cette quadruplicité est sans doute celle de "la négation du droit " donnée par Hegel au \#82 des Grandes lignes de la Pbilosopbie du Droit : «... le droit se rétablit par la négation de sa négation et (. . ) par cetre médiation, il retourne à soi à partir de sa négation et enfin se détermine comme réel et valable, alors que, primitivement, il n'était qu'en soi et quelque chose d'immédiat" (Ph.R., $88 ;$ Pb.D., p. 134). Soulignons que cette intrusion du nombre dans la mobilite dialectique est souvent égarante; qu'il suffise de rappeler que Hegel a divisé la Sciente de la Logique en deux moments : la Logique objective et la Logique subjective. 


\section{LA PRÉSENCE À SOI}

\section{Médiation, négativité, dialectique sont trois notions forgées pour articuler le concept de l'Absolu comme spécula-}

Si l'on envisage la dialectique dans la perspective de la processualité conceptuelle, l'Encydopédie nous enseigne qu'au sens strict elle désigne le deuxième moment de l'activité conceptuclle dont la forme a trois côtés :

(*) le côté abstrait ou relevant de l'entendement,

B) le côté diulectique ou négativement-rationnel,

y) le côté spéculatif ou positinement-rationnel.

(Enz., \#79, $102 ; E n$. , p. 342).

Pour être saisis en leur vérité, ces trois côtés (abstrait, dialecrique et spécularif) ne doivent pas ètre sépatés les uns des autres mais doivent être posés sous la forme réfléchie de "l'unite de l'être-différent et de l'être-non-différent - ou de l'identité de l'identité et de la non-identité " (W, L. I, $59 ;$ S.L. I, p. 63). Traduction modifiée.

Echappant aux chaînes de l'entendement (Verstand), la dialectique appartient en propre a l'ouvre de la raison (Vernunft) pour autant que cette dernière fait intervenir la négation dans la positivité abstraite établic par l'entendement (le coté dialectique ou négativement rationnel) et maintient cette différence dans sa positivité concrète (le côté spécularif ou positivement rationnel. Il faut noter toutefois que dans les Grandes lignes de la Philosophie du Droit, la notion de dialectique recouvre les trois moments: " Le principe moteur du concept, en tant qu'il ne dissout pas seulement les particularisations de l'universel, mais les produit lui-mème, je l'appelle dialectique " (Ph.R., \#31, 47 ; Pb.D., p. 90).

Cette "note technicue " serait incomplète si elle n'indiquait pas qu'il arrive que la raison, fascinée par la "réalité ", renonce au savoir du concept et n'atteigne pas le savoir de la totalité. La réconciliation souveraine de l'être et de la pensée. Il appartient à l'Idée de corriger cette "imputssance subjective de la raison" (W.L., II, 251; S.L., IV, p. 285) à convertir jusqu’à leur transparence l'èrre et la pensée.

L'Idée peur être saisie comme la raison (c'est là la signification philosophique propre du mot "raison"), puis comme le sujet-abjet (. . .) parce qu'en elle sont contenus tous les Rapports de l'entendement, mais dans leur infinie rentrée et identité en eux-mêmes.

(Enz., \#214, $183 ;$ Enc. p. 447)

L.'Idée se distingue de l'entendement et de la raison. À la différence du premier qui isole les déterminités de la penséc en introduisant entre elles une relation extérieure accidentelle ou nécessaire, l'Idée appréhende les déterminités de l'objet comme libres moments d'un tout, les reconnaissant comme apparition de sa vie subjective et objective. L'ldée se distingue de la raison dans la mesure où elle ne se limite pas comme cette dernière à une mise en relation négative (dialectique) ou positive (spéculative) des déterminations singulières mais saisit ces deux côtés de la relation comme moments de l'universalité. Le vestige dualiste qui traine encore dans la raison, I'Idée le dissout en apprehendant l'univers objectif comme étant l'exhibition de sa propre forme universelle. L.'Idée se meut dans la pure transparence de soi :

. . l'idéc n'est donc seulement que dans cette autodétermination, [elle al à sé saisir (zernebmen); elle est dans la pensée pure, où la différence n'est pas encore un être-autre, bien plutôt elle est et reste parfaitement transparente pour elle-mème. » (W.L., II, 485 ; S.L., IV, p. 550). Traduction modifiée.

Indiquons pour finir que comme "travail du négatif ", la dialectique se déploie différemment selon les niveaux de progression de la processualité conceptuelle (de l'Être jusqu'à l'Idée) et selon un mode d'opération propre à chacun de ces niveaux.

La forme abstraite de la progression est dans l'être un autre et un parsage dans un autre, dans l'essence un parattre dans l'opposé, dans Je concept la différenciation du singulier d'avec l'unitersalité qui se continue comme telle dans ce qui est différencié d'elle, et qui est en tant quidentite avec lui " (Enz., \#240, 195 ; Enc., p. 461$)$. 
tion - restitution de l'identité (le Même) dans la Différence (l'Autre : dédoublement du Même) - et subvertir par là les visées très traditionnelles du savoir selon lesquelles le vrai trouverait son site dans une correspondance entre un sujet connaissant (concept) et un objet qui lui est extérieur (étant). C'est ce schème que veulent faire éclater les notions de médiation, de négativité et de dialectique ordonnées à penser la réversibilité des termes concept et être.

Nommons-nous le savoir le concept, nommons-nous, d'autre part, l'essence ou le vrai l'étant ou l'objet, l'examen consiste alors à voir si le concept correspond à l'objet ${ }^{52}$.

Ce qui est en cause dans ce texte, ce n'est pas seulement une théorie de la connaissance (toute théorie de la connaissance ne concerne que la connaissance de la connaissance), mais une révolution de la connaissance ou du savoir comme tel : la connaissance est être et l'être est connaissance. C'est cette unité du concept et de la réalité qui vibre dans les trois textes choisis plus haut (cf. p. 9). Relisons celui qui ouvre l'Introduction à la Phénoménologie de l'Esprit :

C'est une représentation naturelle que, avant que l'on en vienne, dans la philosophie, à la Chose même, savoir au connaître effectif de ce qui est en vérité, il serait nécessaire de s'entendre auparavant sur la connaissance qu'on considère comme l'instrument à l'aide duquel on s'empare de l'absolu ou comme le moyen grâce auquel on l'aperçoit. Une telle préoccupation semble justifiée, en partie, parce qu'il pourrait y avoir diverses espèces de connaissances, et que dans le nombre l'une pourrait être mieux adaptée que l'autre pour atteindre ce but final, - justifiée donc aussi par la possibilité d'un choix erroné parmi elles, - en partie aussi parce que la connaissance étant une faculté d'une espèce et d'une portée déterminée, sans détermination plus précise de sa nature et de ses limites, on peut rencontrer les nuées de l'erreur au lieu d'atteindre le ciel de la vérité. À la fin, cette préoccupation doit se transformer en la conviction que toute l'entreprise de gagner à la conscience ce qui est en soi par la connaissance est dans son concept un contre-sens, et qu'il y a entre la connaissance et l'absolu une ligne de démarcation très nette.

52. Ph.G., $71 ;$ Ph.E. I, P. 73. 
Quelles sont les démarches philosophiques visées ici par Hegel ? Il s'agit en réalité de toutes les philosophies modernes depuis Descartes, qui ont prétendu que si la philosophie veut s'installer en terre ferme, elle ne doit rien présupposer d'autre que le fait de connaitre et dire d'abord ce qu'est la connaissance et comment il faut la connaître. Le texte que nous venons de relire fait état de deux conceptions de la connaissance. La première, celle qui envisage la connaissance comme un instrument actif, a été élaborée par Kant (l'entendement est une faculté qui produit des concepts), la seconde, celle qui considère que la connaissance est un milieu passif, a été développée dans l'empirisme, principalement dans la philosophie de Hume (connaître, c'est subir des impressions). Ces démarches, qui ne veulent rien présupposer, s'appuient en fait sur des concepts contestables: elles postulent, selon Hegel, une ligne de démarcation entre l'Absolu et la faculté de connaître. Cette observation de Hegel constitue une critique subtile : en reprochant aux démarches qu'il rejette d'introduire cette ligne de démarcation que bien sûr il ne porte pas à son compte, Hegel introduit lui-même un terme étranger aux problématiques qu'il discute : l'Absolu. Critique d'autant plus subtile que c'est à travers une certaine répétition de la philosophie kantienne que s'est instaurée la pensée hégélienne du procès spéculatif.

On sait que le criticisme kantien, même lorsqu'il exalte l'Apparaître, n'en fait que le substitut de l'Être: sa vérité, l'Apparaître ne la reçoit pas de l'Être (l'Erscheinung ne s'identifie pas au $S$ cbein) mais de l'esprit fini qui atteint par là à « une immense humilité de l'esprit, (. . .) ne rien faire reposer sur le connaître ${ }^{53}$. Cette humilité, Foi et Savoir l'avait qualifiée d'« indigne résignation $»^{54}$, y décelant même le signe de la « jubilation qu'éprouvent l'entendement et la finitude à s'être décrétés comme absolus ${ }^{55}$. Comment Hegel interprète-t-il l'enquête transcendantale menée dans la Critique de la Raison Pure? À ses yeux, on méconnaîtrait l'intention véritable de cette enquête si on en réduisait la problématique à « la

53. G.Ph., I, 343 ; L.H.Ph. I, p. 152. Traduction modifiée.

54. G.W., $336 ; F . S .$, p. 217.

55. G.W., $338 ; F . S$, . . 219 
connaissance des phénomènes comme l'unique mode de connaître " ${ }^{56}$. C'est bien plutôt sur une "idée vraiment spéculative ${ }^{57}$ que s'appuie, selon Hegel, la démarche kantienne. En témoigne la structure de l'imagination transcendantale où surgissent, mutuellement accordés, l'intuition et l'entendement. Lisons Hegel :

On ne peut rien comprendre de toute la déduction transcendantale tant des formes de l'intuition que de la catégorie en général, sans distinguer du Moi en activité de représentation, du Moi-sujet que Kant appelle le simple accompagnement de toutes les représentations, ce qu'il appelle le pouvoir de l'unité originaire synthétique de l'aperception, sans reconnaître cette imagination, non comme intermédiaire simplement introduit entre un sujet et un monde ayant tous deux une existence absolue, mais comme ce qui est premier et originaire ${ }^{58}$.

Le sens de ce passage n'est pas douteux. Si, pour Hegel, le mérite de Kant a été de s'approcher du principe de la spéculation absolue - dans la structure de l'imagination transcendantale, « l'identité est (. . .) parfaitement identique à la différence ${ }^{59}-$, son seul tort fut de préférer s'en détourner pour que la philosophie devienne « de nouveau servante d'une foi $"{ }^{60}$. Tel est le destin des philosophies de la réflexion: pressenti comme "richesse infinie ", l'Absolu prend le visage imprécis de la nuit où vont s'échouer les oppositions fixées par l'entendement, l'Absolu est le néant « d'où est sorti tout l'être, toute la diversité du fini ${ }^{61}$.

Lorsque, dans la troisième étude ("La réflexion comme instrument de l'activité philosophique ») de son Mémoire sur la Différence, Hegel examine cette équivalence entre le "néant " et la " richesse infinie ", il affirme que les premiers chemins tracés par la connaissance réflexive mènent pourtant à une détermination fondamentale de l'Absolu. Si l'Absolu "disparaît dans la nuit ", si, à la suite des mises en position de l'entendement, il se présente comme négation des êtres

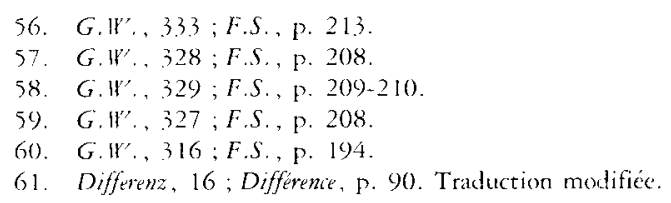


déterminés et comme "Absolu négatif ", il n'en est pas moins affirmé comme l'unité d'où émane la multiplicité de l'être. Cette affirmation de la simple réflexion de l'entendement demeure pourtant indifférenciée, ou pour ainsi dire irréfléchie, dans la mesure précisément où l'identité de l'Absolu n'est pas établie comme faisant retour à elle-même dans la multiplicité de l'être où elle est pourtant pressentie. Convertir cette différence entre l'unité indéterminée de l'Absolu et la multiplicité déterminée de l'être en une autodifférenciation de l'Absolu, saisir cette autodifférenciation comme l'acte par lequel l'Absolu dans un même mouvement se révèle à lui-même et retourne à lui-même, telle est l'affaire propre de la réflexion devenue authentiquement philosophique, telle est la nécessité qu'elle porte "dans son travail, en tant que spéculation » (in ihrem Geschäfte, als Spekulation) » ${ }^{62}$.

La philosophie spéculative dépouille ainsi de son caractère de radicalité « le problème de la connaissance»: elle révèle que ce qui vient en question ou ce qui est en litige dans ce problème, c'est, plus profondément encore que l'Apparaître de l'Absolu qui veut « être en soi et pour soi depuis le début près de nous ${ }^{63}$, le fondement même de cette automonstration de l'Absolu dont Hegel nous dit qu'il est non seulement «pur anéantissement (. . .) de la finitude ${ }^{64}$ et « source (. . .) de la finitude ${ }^{65}$, mais le «pays d'origine » de la vérité d'où elle s'élève « comme d'un abîme mystérieux ${ }^{66}$. Qu'est-ce à dire sinon que la connaissance porte en elle une non-connaissance, qu'une non-vérité est inscrite au cour même de la vérité, qu'il y a un espacement dans la manifestation, un voilement dans le dévoilement?

S'il est clair que de nombreux textes laissent entrevoir que le discours hégélien n'a cessé de se mouvoir dans cet horizon, il parait pourtant incontestable que dans le mouvement même où il scrute ce retrait de l'origine, Hegel le dissimule en l'acheminant vers la présence absolue de soi à soi. Ainsi, loin

\footnotetext{
62. Differenz, 16; Différente, p. 90. Traduction modifiée.

63. Ph.G., $64 ;$; Ph.E., I, P. 66.

64. G.W. $413 ;$ F.S., p. 298.

65. G.W., 413;F.S., p. 298.

66. G.W. $413 ;$ F.S., p. 298.
} 
d'être soustrait à toute coïncidence, l' « abîme mystérieux, pays d'origine" de la vérité est intégré dans le champ de la manifestabilité logique de tout ce qui est : il n'est, comme l'écrit Hegel, que "le moment de l'Idée suprême, mais pas davantage $"{ }^{67}$. Et de même l'anéantissement indissociable de ce mystérieux abîme n'est qu'un côté de l'Idée absolue dont Hegel, s'inspirant du livre XII de la Métaphysique d'Aristote, dira dans le dernier paragraphe de l'Encyclopédie qu'elle «se met-en-action, s'engendre et jouit éternellement de soi comme Esprit absolu (sich ewig als absoluter Geist betätigt, erzeugt und geniesst) ${ }^{68}$.

Que ce schème de la coïncidence de soi avec soi ne régisse pas que les écrits dits systématiques, mais guide tout l'itinéraire hégélien, on peut le remarquer à propos de la méditation constante du thème de l'amour. Ainsi le Tübinger Fragment présente l'amour comme ce qui « se trouve soi-même dans d'autres hommes ou plutôt s'oubliant soi-même s'échappe de son existence, vit pour ainsi dire dans les autres, $[y]$ sent et [y] agit ${ }^{69}$. Les Leçons sur la philosophie de la Religion reprennent la même conception :

L'amour différencie deux êtres qui l'un pour l'autre ne se distinguent pas. Avoir conscience, avoir le sentiment de cette identité, d'être hors de moi-même et dans l'autre, voilà l'amour ; ma conscience n'est pas en moi, mais dans l'autre; cet autre en lequel seul j'ai ma satisfaction et la paix avec moi-même - et je ne suis que si je suis en paix avec moi-même ; si je ne la possède pas, je suis la contradiction qui se divise, - cet autre, également hors de lui-même, a en moi seul sa conscience et tous deux nous ne sommes que cette conscience de notre extériorité et de notre identité, cette intuition, ce sentiment, ce savoir de l'unité. Voilà l'amour et ce sont de vaines paroles si l'on parle de l'amour sans savoir qu'il est la différenciation et la mise de côté (aufheben) de la différence -70 .

Autant ces présentations sont étrangères au ressentiment, autant elles ignorent l'inquiétude du retrait. Loin de signaler un désarroi éveillé par le rayonnement de la rencontre, l'amour

67. G.H. . 414;F.S. p. 298.

68. Ens., \#463, 463 ; Enc., (Gandillac), p. 500.

69. T.F., N., 18.

70. V.Ph.R., II, $227 ;$ L.Ph.R., III, p. 77. 
naît d'une connaissance qui sait se préserver de tout dépaysement. Disposition à l'accueil, le mouvement de l'amour n'est qu'une attente sans frémissement qui, loin de se réserver devant l'énigme de ce qui se prodigue, ouvre un espace transparent où vibrent ensemble le ciel et la terre dans un monde apaisé. Exalté comme ravissement et "jouissance pleine de vitalité (vollblütiger Genuss) » ${ }^{71}$, l'oubli de soi dans l'autre n'est qu'un moment d'une jubilation absolue.

Hegel se plaisait à se réclamer d'Héraclite : "il n'y a pas une proposition d'Héraclite que je n'aie reprise dans ma Logique $^{72}$. "Cette filiation protège-t-elle la parole inspirée de l'Obscur Penseur d'Éphèse : «La nature aime à se cacher "?

Département de Philosophie Université de Sherbrooke

Liste des ouvrages consultés

BIRAULT, H., "L'onto-théo-logique hégélienne et la dialectique", in Tijdschrift vonr Philusophice, 4, décembre 1958, p. 646-723.

BLOCH, E., Sujet-Objet. Eilairitements sur Hegel. Paris, Gallimard, 1977.

BOURGEOIS, B., La pentée politique de Hegel. Paris, P.U.F., 1969.

---- Hegel à Francfort on Judaisme - Christianisme - Hégélianisme. Paris, Vrin, 1970.

---- «Présentation " de la trad. de HEGEL, Encyclopédie des sciences philosophiques. 1.- La science de la lngique. Paris, Vrin, 1970, pp. 7-109.

HYPPOLITE, J., Genese et struture de la Phénoménolngie de l'Esprit de Hegel. Paris, Vrin, 19)39-1941.

----- Introduction à la philosopbie de lbistoive de Hegel. Paris, Rivière, 1948.

---- Logrique et Existence. Essai sur la logique de Hegel. Paris, P.U.F., 1961

....- "Hegel", in Figures de la pensée philosophique, Paris, P. U.F., 1971.

JANICAUD, D., Hegel et le destin de la Grese. Paris, Vrin, 1975.

LABARRIÈRE, P.-J., Strutures et montement dialectique dans la Pbénoménologie de l'Esprit de Hegel, Paris, Aubier, 1968.

-...- Introduction à une lecture de la Phénoménologie de lEsprit. Paris, Aubier, 1979.

---- "Le concept hégélien, identité de la mort et de la vie ", in Archives de philosiphic, 33, 1970, p. 579-604.

----- "La Phénoménologie de l'Esprit comme discours systématique: Histoire, Religion et Science ", in Hegel-Studien, Bd. 9, p. $131-153$

LEBRUN, G., La patience du concept. Fssai sur le discours bégélien. Paris, Gallimard, 1972.

PEPERZAK, A.-T.-B., Le jetme Hegel et la tision morale do monde. La Haye, Nijhoff, 1969.

71. T.F., N., 6.
72. G.Ph., I. 344;L.H.Ph., I, p. 154. 
POGGELER, O., Hegels Idee einer Phänomenologie des Geistes. Freiburg/München, Alber-Broschur Philosophie, 1973.

Hegel. Freiburg/Muichen, Alber-Kolleg Philosophic, 1977.

TAMINIAUX, J., La mostalgie de la Grese à laube de l'iäalisme allemand. Kant et les Gres dans litinérative de Sibiller. de Hölderlin et de Hegel. La Haye, Nijhoff, 1967.

"Introduction" de la trad. de HEGEL, Systeme de la tie éthique. Paris, Payot, 1976.

---- Le regard et l'exédent. La Haye, Nijhoff, Phanomenologica, 1977. 